
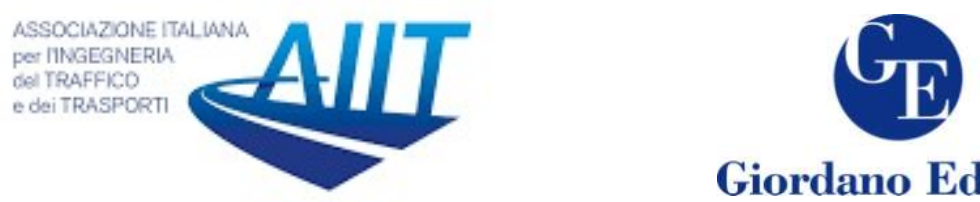

Giordano Editore

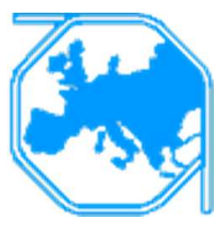

\title{
Lambert W Based Speed Reduction Model in Presence of Pedestrian Movements: Case Studies on Undivided Streets
}

\author{
Sourabh Thakur ${ }^{1}$, Sraddha Maurya ${ }^{1}$, Satish Chandra ${ }^{2}$, Subhadip \\ Biswas $^{1 *}$ \\ ${ }^{1}$ Department of Civil Engineering, National Institute of Technology Hamirpur, Hamirpur-177005, \\ Himachal Pradesh, India \\ ${ }^{2}$ CSIR-Central Road Research Institute, Delhi-Mathura Road, New Delhi-110025, India
}

\begin{abstract}
Pedestrian movements sharing right-of-way with vehicular traffic have adverse impacts on the mobility of the traffic stream. Pedestrian movements both along and across the road often force drivers of approaching vehicles either to stop completely or to slow down and change the existing lane. It ultimately results in a decrease in stream speed. With the aim of determining the influence of pedestrian movements, the present study collected traffic data at a standard section (without pedestrian movements) and both traffic and pedestrian data at a pedestrian section (with considerable pedestrian movements). To determine the speed at the standard section, this paper presents a novel 'Lambert $\mathrm{W}$ function'-based speed prediction model in the context of a two-lane undivided urban road. When stream speeds of the pedestrian section were compared to the stream speeds obtained through the speed prediction model at the same traffic volume condition in absence of pedestrians, a significant reduction was observed. This reduction in stream speed is governed by pedestrian parameters. A new pedestrian parameter 'lateral position of pedestrian from the edge while walking along the road' was conceived in this study along with few other parameters to predict Percent Speed Reduction (PSR). Intensities of these pedestrian parameters were observed varying over time which results in a high fluctuation in PSR within a range of $29 \%$ to $62 \%$. Finally, this investigation forwards an empirical model of Percent Speed Reduction (PSR) to predict the stream speed in the presence of onstreet pedestrian movements on undivided urban roads. The outcome of this paper will help transport planners to estimate the efficiency of pedestrian infrastructure projects before implementation.
\end{abstract}

Keywords: pedestrian-vehicle interaction; speed modeling; pedestrian movements; Lambert W function; urban roads.

\section{Introduction}

Walking is considered as an elementary mode of transportation. Although other modes of transportation are used frequently, yet most of the trips of an individual start and end are being pedestrian. Pedestrians are prime road users and they move either along the road or across the road. In most of the developing countries, the absence of sidewalk often

\footnotetext{
${ }^{*}$ Corresponding author: Subhadip Biswas (subhadipbiswas.in@gmail.com)
} 
forces pedestrians to share the road space with vehicular traffic on urban roads. In fact, when the sidewalk is having distressed portions (like, cracking, patching, pothole etc.) (Corazza, Di Mascio and Moretti, 2018) or partially occupied by street-hawkers (Sukhadia et al., 2016) the majority of pedestrians do not prefer to access the sidewalk. Instead, they walk on the carriageway and share space with other motorized vehicles. Interaction between pedestrian and vehicular movements is inconsistent in nature as it varies depending upon the magnitude of individual volume levels (Chen and Wang, 2015). When pedestrians walk along the road are in considerable volume, vehicles shy away towards the center of the road ensuring sufficient lateral distance from pedestrians. Thus, it narrows down the effective carriageway width available for vehicle movements and eventually demotes the mobility condition of the road. On the other hand, for pedestrian cross-movements, they often prefer jaywalking and cross at any point of the road as per their convenience specifically for undivided urban streets of developing countries (Hawas and Khan, 2012). Pedestrians decide to cross the road in their first attempt and take the shortest route possible. The crossing time is governed by many factors such as the pedestrian's gender, age, cell phone use, style of clothing, crossing in a group, crossing direction, the presence of an approaching vehicle, etc. (Shaaban, Muley and Mohammed, 2018). This increases the potential of vehicle-pedestrian conflict and thus the safety of pedestrians is compromised. Pedestrian crossing styles have a significant impact on driver's behavior. Moreover, pedestrians approaching to cross the road force drivers to reduce their speed (sometimes to stop completely) or to change their lane to avoid a conflict incident. Again, this finally results in the reduction in stream speed. Prediction of stream speed under the influence of on-street pedestrian movements holds significance since it is one of the major factors based on which a decision is taken whether a pedestrian facility would be implemented or not. A speed reduction model would be an answer to the question 'How much Level-of-Service (LOS) of an urban road segment will improve if a pedestrian facility, say underpass, is constructed?'. This is the reason behind a good volume of the studies conducted already to quantify the speed reduction due to the presence of on-street pedestrian movements. The present study revisited the past research related to the impact of pedestrian movements on vehicular traffic. Few studies (Adinarayana and Anil, 2017) observed the reduction in stream speed on urban roads under the influence of substantial pedestrian volume along the road. However, no quantification of this reduction was reported. Bassani et al. (Marinelli, Dalmazzo and Bassani, 2013) estimated a decrease of $7.23 \mathrm{~km} / \mathrm{h}$ in the stream speed when pedestrians were sharing the right-of-way with vehicular traffic. Apart from 'along the road' movements, the significant effect of pedestrian cross-movements on the mobility of traffic stream was thoroughly reported in the literature. Varhelyi (Várhelyi, 1996) observed that stream speed is decreased by $2 \mathrm{~km} / \mathrm{h}$ when the pedestrian was about to cross from one side of the undivided road. However, in case of pedestrian crossing from both sides of the road, there was a decrease of $5 \mathrm{~km} / \mathrm{h}$ in stream speed. Similarly, decrease of $7.7 \mathrm{~km} / \mathrm{h}$ (Kadali, Chiranjeevi and Rajesh, 2015)and $2 \mathrm{~km} / \mathrm{h}$ (Thiessen, El-Basyouny and Gargoum, 2017)in the stream speed were witnessed at the mid-block pedestrian crossing on divided urban roads. However, all aforementioned works share a common limitation of considering 'pedestrian movement' as binary variable. Neither these studies estimated stream speed under varying intensity of pedestrian movements nor any adjustment factor or empirical model developed.

In this regard, some researchers extended the investigation further to examine how this influence varies with the change in intensity of pedestrian movements. An empirical 
model developed by Shukla et al. (Salini et al., 2016) suggests that with the addition of each new pedestrian walking along the road, there will be a decrease of $0.35 \mathrm{~km} / \mathrm{h}$ in the stream speed. A study conducted by Hawas and Khan (Hawas and Khan, 2012) used the Fuzzy technique to show graphically the reduction in vehicular speed with the increase in the pedestrian cross volume. Aronsson and Bang (Aronsson and Bang, 2006) suggested a speed model to determine the average traffic speed on divided urban roads considering pedestrian cross volume and traffic volume as design variables. Munawar (Munawar, 2011)investigated the influences of side-friction factors on traffic speed. 'Pedestrian cross volume' was considered as one of the major side-friction parameters along with the number of stopping buses, parked vehicles, entry vehicles into the street and heavy vehicles. On the other hand, Golakiya \& Dhamaniya (Golakiya and Dhamaniya, 2019) developed a speed model to estimate the influence of pedestrian cross volume on individual vehicle category in a mixed traffic stream. The study witnessed the reduction in the average speed of all vehicle categories excluding heavy vehicles when the pedestrian cross volume increases on the road. However, to use the model, a user needs to follow an iterative method which can be considered as a drawback of this model from the field application perspective.Hence, after reviewing the former studies conducted on this topic, some gaps in the research are identified and discussed in the follwing section.

\section{Problem formulation and research gaps}

Stream speed is a key parameter for the assessment of LOS and travel time reliability on an urban road network. Thus, the impact assessment of pedestrian movements on the mobility of the traffic stream holds significance since the relationship between these two factors is a prerequisite for the performance assessment of an urban corridor. Due to these utilities, a number of investigations were already carried out towards developing the efficient empirical model or adjustment factor in order to predict the stream speed in the presence of on-street pedestrian movements. However, after going through these previous studies, the following research gaps were identified and the directions of further investigations were decided accordingly.

Majority of the previous attempts (Várhelyi, 1996), (Marinelli, Dalmazzo and Bassani, 2013), (Adinarayana and Anil, 2017) considered 'pedestrian movements' as a binary variable; either 'absent' (0) or 'present' (1) and accordingly estimated the reduction in stream speed owing to the presence of on-street pedestrian movements. The role of 'intensity of pedestrian movements' was ignored in these studies. However, in a typical urban road scenario, the intensity of pedestrian movements is not constant rather it fluctuates widely with time. The change in intensity of pedestrian movements may lead to the change in the reduction of stream speed.

Divided urban roads are expected to be more regulated and pedestrians can only cross the road through median openings provided at designated locations as shown in Figure 1. In addition, vehicles running on median lanes of divided urban roads are marginally affected by the pedestrians walking along the road. 


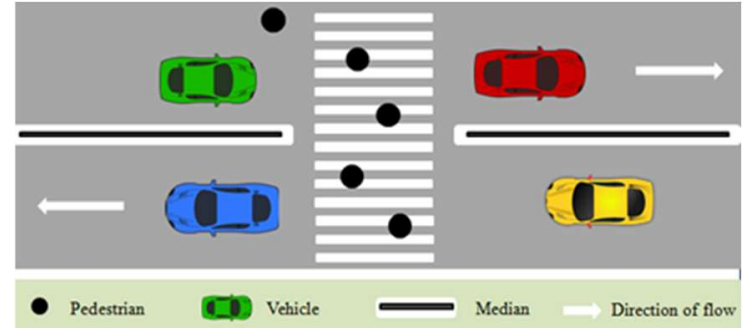

(a)

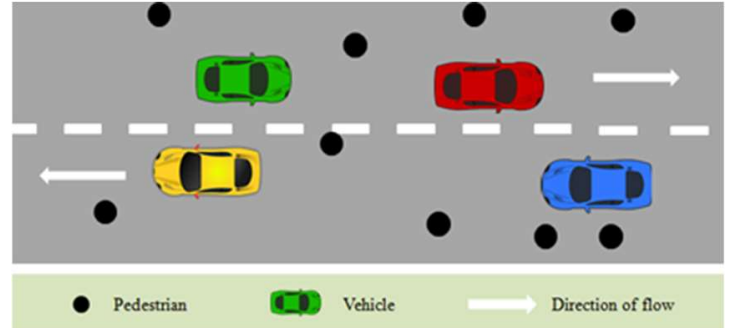

(b)

Figure 1: Pedestrian movements on a) Divided road b) Undivided road

On the other hand, undivided urban roads are those which do not have any kind of median to separate opposite directional traffic. Undivided urban roads generally come under the 'collector' or 'local street' category (IRC: 106-1990). On such roads, the influence of pedestrian movements is more predominant as they often cross the road at any undesignated location resulting in a more acute reduction in the stream speed. However, the majority of the previous studies (Kadali and Tadi, 2015) were contextual to divided urban roads only. Despite the fact that pedestrian movements are more unrestricted and predominant on undivided urban roads, little emphasis was given on this type of road.

All of the previous studies considered on-street pedestrian movements either 'along the road'(Advani and Nisha, 2013), (Munawar, 2011)'across the road' (Golakiya and Dhamaniya, 2019), (Jin et al., 2013) while estimating the impact of pedestrian movements on the reduction in stream speed. In spite of the fact that pedestrians move along and across the road simultaneously, none of the previous attempts analyzed the combined influence of both along and across pedestrian movements on the mobility of urban traffic.

In estimating the impact of pedestrians moving along the road on the stream speed, the previous studies (Salini, George and Ashalatha, 2016), (Adinarayana and Anil, 2017) considered a single parameter i.e. 'pedestrian volume along the road' $\left(\mathrm{P}_{\mathrm{al}}\right)$. In this regard, the lateral position of pedestrians across the carriageway width while walking along the road holds significance in influencing the movements of vehicular traffic. A lateral shift of pedestrians towards the center of the road reduces the effective carriageway width available for vehicle movements. As a result, vehicles move at lower speed while negotiating a reduced effective carriageway width. Conversely, a lateral shift of pedestrians towards the edge of the carriageway is expected to increase the stream speed. The aspect of the lateral position of pedestrians was not addressed in previous research.

Hence, on this background, the present research aims i) to identify suitable pedestrian parameters which have significant influences on the mobility of through traffic and ii) to develop a speed prediction model based on the shortlisted pedestrian parameters on undivided urban roads. The present study captures the combined influence of pedestrian movements both along and across the road and also addresses all aforementioned research gaps. 


\section{Research methodology}

Present analysis and the outcome of the study are entirely based on the field data collected at different sections of undivided urban roads. To select a potential study location suitable for this investigation, the following criteria were considered.

- Section must be straight with leveled longitudinal gradient.

- Section should not have bus stops and on-street parking activities.

- Section should be located adequately away from the neighboring intersection warranting nominal impact of intersection on the mid-block traffic movements.

- Surface conditions of the road must be good without any pavement distress.

Locations identified for the present study were classified into two categories;

(a) Standard section (S-section) having no or minimal pedestrian movements and

(b) The pedestrian section (P-section) having considerable pedestrian movements along and across the road.

A videography survey was used to collect traffic data for S-section and traffic along with pedestrian data for P-section. Then, the video files were taken to the laboratory to extract the information required for the analysis. Vehicles were classified into a number of categories based on observation. Classified traffic speed and classified traffic volume data were extracted for each 5-minute time interval. Later, classified traffic volumes were transformed into their homogeneous equivalent using Passenger Car Unit (PCU) values recommended in Indo-HCM (CSIR- Central Road Research Institute, 2017). Under pedestrian data, (a) pedestrian volume along the road, (b) pedestrian volume across the road and (c) lateral position of pedestrian from the edge while walking along the road were extracted for the same time interval exclusively for P-section. In the absence of pedestrian movements, traffic speed on S-section is entirely governed by the traffic volume. In developing a volume-based speed model, the unknown variable (speed in this case) appeared on both outside and inside of a logarithm function in an equation. Hence, 'Lambert W' function was adopted to solve the equation and to develop the speed model. The developed model can predict the speed of the traffic stream for a given traffic volume condition. Therefore, the traffic speed observed at the P-section was compared with the traffic speed obtained through the Lambert W-based speed model at the same traffic volume condition. As it was expected that the traffic speed for P-section would be lower as compared to the same of S-section, 'Percent Speed Reduction' (PSR) was estimated for each 5-minute time interval. Again, the degree of reduction in speed was expected to change with the variation in the intensity of pedestrian movements prevailed during the observation time. Prior to considering all pedestrian parameters as input variables in modeling PSR, the interdependency of these parameters among each other was checked by developing the correlation matrix. Based on the correlation results, pedestrian parameters were shortlisted and considered for the next phase of analysis. Since the pedestrian parameters as design variables have diverse units and scales, the 'Feature Scaling' method was adopted to convert the design variables as unitless and into a common scale of 0 to 1 . Finally, these scaled pedestrian parameters were used in the method of least squares to achieve the objective of developing the speed reduction model. The research methodology discussed above is also shown in Figure 2 in form of a flowchart. 


\section{Field data collection}

\subsection{Collection Procedure}

Based on the selection criteria discussed in Section 3, two road sections (one S-section and one P-section) were chosen for field data collection in two different cities of India namely, Dehradun and Kolkata. These road sections were chosen intentionally from two different cities so that the outcome of this study would be more generic and non-cityspecific. These two road sections are identical and are on two-lane undivided urban roads of the same 5.5-meter carriageway width. Other details of these sections and the duration of field data collection are given in Table 1.

Table 1: Details of data collection

\begin{tabular}{|c|c|c|c|c|c|}
\hline S. No. & Name of the Road & City & Type of section & $\begin{array}{l}\text { Duration of data } \\
\text { collection }\end{array}$ & Day \\
\hline 1 & Cross Road & Dehradun & S-section & $7 \mathrm{AM}$ to $11 \mathrm{AM}$ & Weekday \\
\hline 2 & $\begin{array}{l}\text { Picnic } \\
\text { Road }\end{array}$ & Kolkata & P-section & $7 \mathrm{AM}$ to $11 \mathrm{AM}$ & Weekday \\
\hline
\end{tabular}

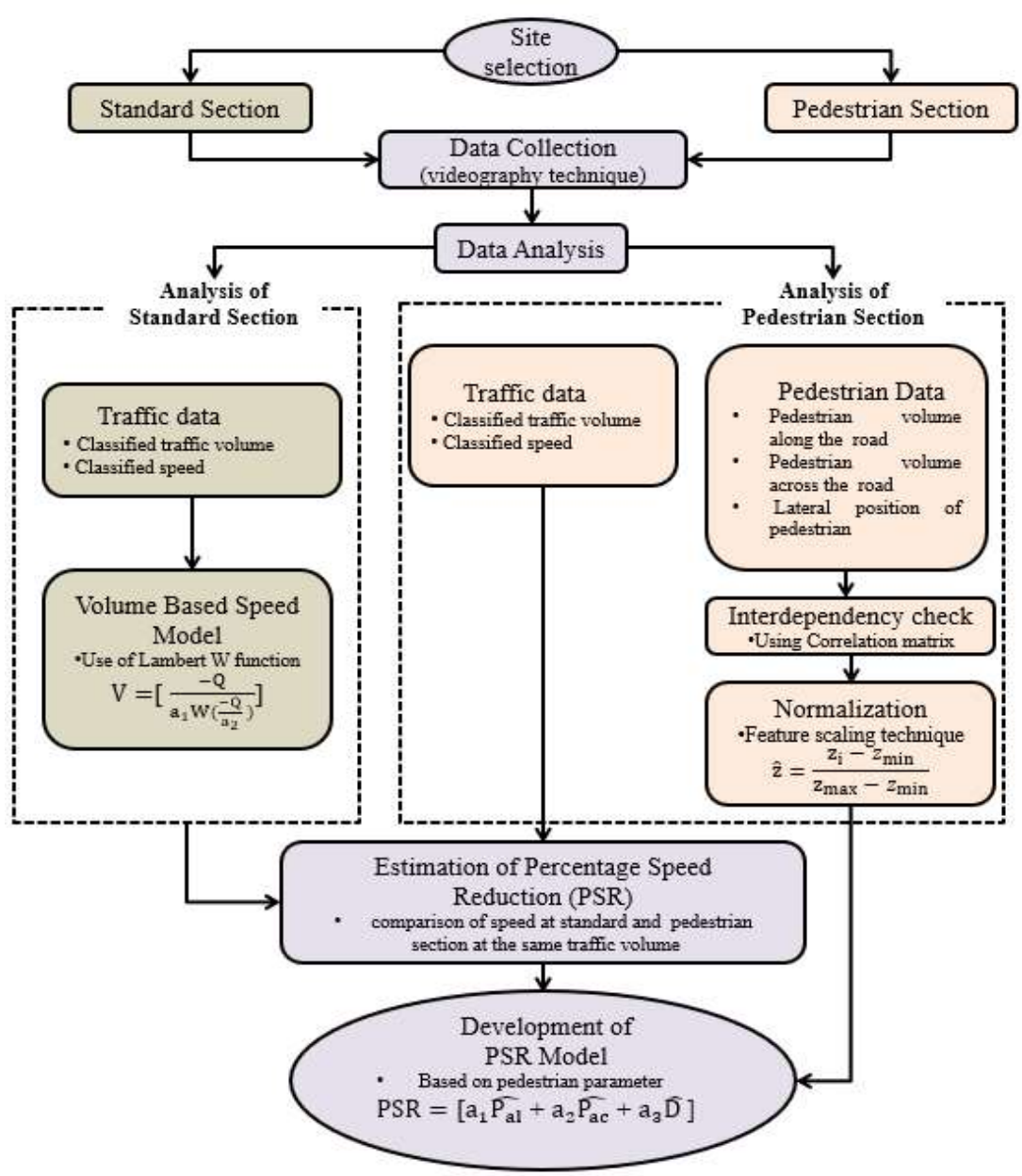

Figure 2: Flow-chart showing research methodology

To collect both traffic and pedestrian data, a high-resolution camera was mounted on a $4.5 \mathrm{~m}$ tall camera stand. The entire set-up was kept roadside in such a manner so that it can capture the pedestrian (only for P-section) and the traffic movements (for both 
sections) uninterruptedly on the selected road section. Videography was conducted from 7 AM to 11 AM on weekdays in normal weather conditions. The observation period covers both off-peak and peak hours of traffic. The entire set-up of the videography survey is shown in Figure 3.

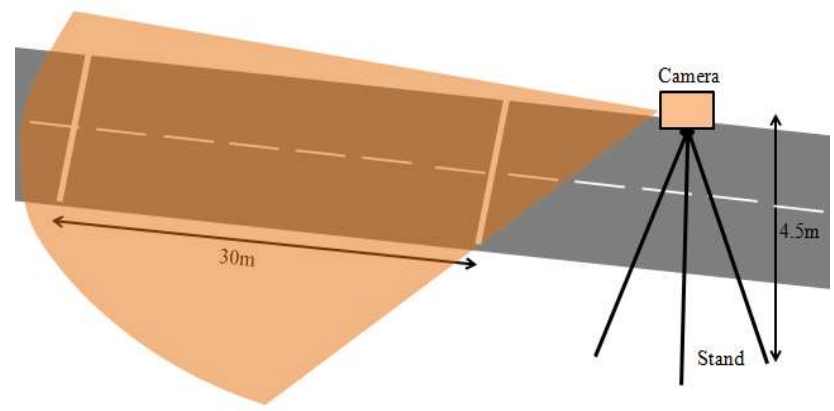

Figure 3: Arrangement of videography survey

Vehicles were classified into six categories; Two-wheeler; Three-wheeler; Car; Heavy vehicle; Bicycle and Pedal Rickshaw. A longitudinal trap length of $30 \mathrm{~m}$ was marked on each study location using virtual lines as shown in Figure 4a. Classified traffic volumes for each 5-minute time interval were extracted by counting the number of vehicles under individual categories crossing the road section.

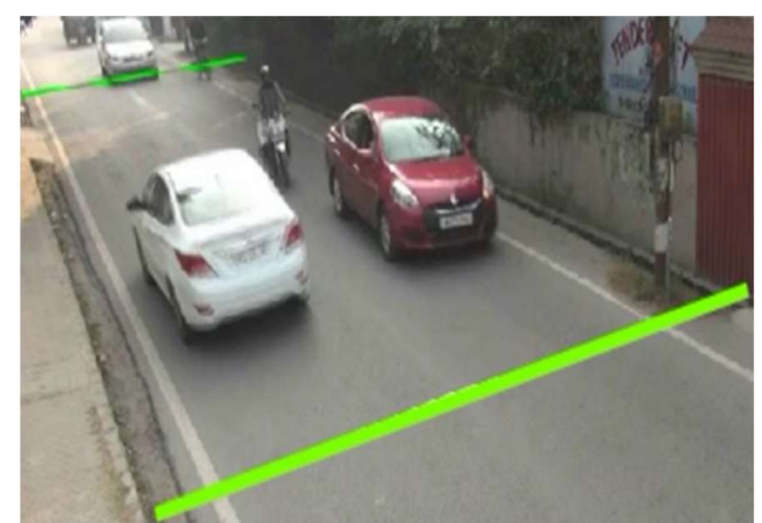

(a)

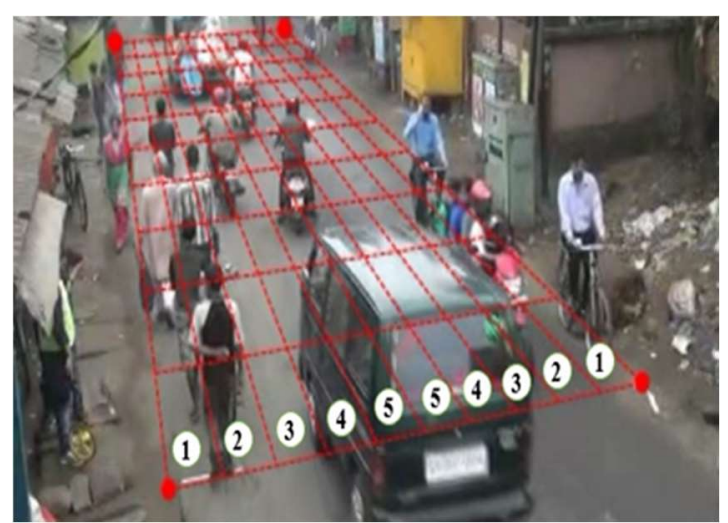

(b)

Figure 4: Data extraction on a) standard section and b) pedestrian section

Entry time into the segment and exit time from the segment were recorded for the individual vehicle with an accuracy of 0.01 second. The speed of each vehicle was calculated based on the time (difference between the entry and the exit time) taken by the vehicle to cover the trap length of $30 \mathrm{~m}$. During extracting pedestrian data, it was realized that the position of the pedestrian while walking along the road, may have a significant influence on vehicular speed. Pedestrians in India are often observed not to follow the edge of the carriageway while walking along the road and sharing space with vehicular traffic. Pedestrians with faster walking speeds generally tend to shift laterally more towards the center of the road. This behavior not only causes additional risks towards conflict with motorized vehicles, the mobility on the road is also compromised. Although the previous studies did not consider the aspect of the lateral position of pedestrians in impact assessment, the present study primarily includes this aspect and further examines the significance of it in influencing the stream speed. Therefore, in order to track the 
position of pedestrians, the carriageway width of the P-section was divided into ten segments having equal width of $0.55 \mathrm{~m}$. Segment adjacent to the edge of the carriageway was denoted as 'Segment 1'. The rest of the segments were denoted in ascending order 'Segment 2-5' towards the center of the road as shown in Figure 4b. Segment number $\left(\mathrm{S}_{\mathrm{N}}\right)$ followed by each pedestrian while walking along the road was recorded. The concept of 'lateral position of the pedestrian from the edge while walking along the road' is illustrated in Figure 5.

The lateral position (D) of the pedestrian from the edge of the carriageway was calculated using Equation (1).

$$
\mathrm{D}=\frac{\mathrm{S}_{\mathrm{N}} \times \mathrm{W}}{\mathrm{N}}
$$

Where, $\mathrm{W}=$ Carriageway width (m) $\mathrm{N}=$ Total number of segments

Lastly, pedestrian volumes both along and across the road were extracted by counting the number of pedestrians while playing the video files.

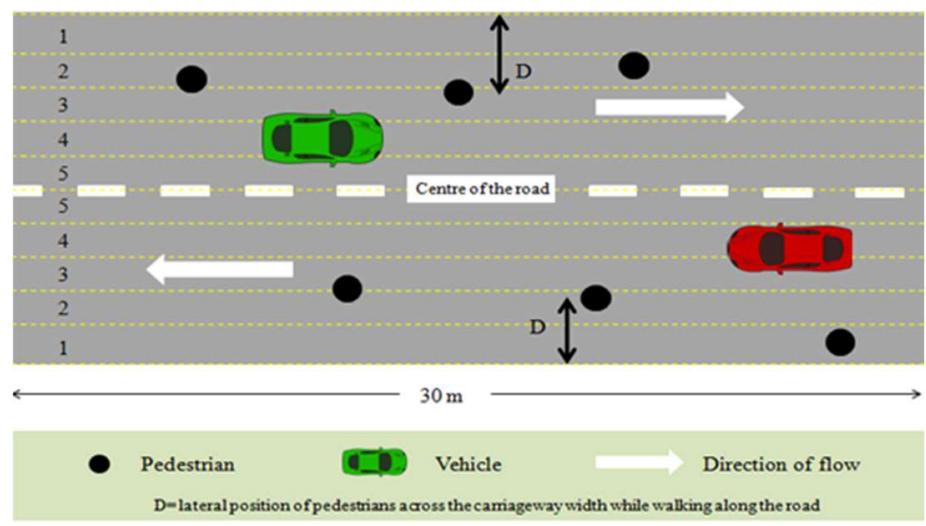

Figure 5: Extraction of lateral position data for the pedestrians walking along the road

\subsection{Observed Traffic and Pedestrian Data}

A high degree of heterogeneity composed of six vehicle categories (viz. motorized two-wheelers, three-wheelers, cars, heavy vehicles, bicycles and pedal rickshaw) was observed at both S-section and P-section. However, in both cases, the two-wheeler as a category held the major share in traffic composition. Classified traffic volumes extracted from the video files, were converted to homogenous equivalent (PCU/h) as discussed in Section 3. Variation in traffic volume observed in both sections is exhibited in Figure 6. Both directional traffic volumes for S-section were varying from $395 \mathrm{PCU} / \mathrm{h}$ to 1046 $\mathrm{PCU} / \mathrm{h}$ while it was varying from $913 \mathrm{PCU} / \mathrm{h}$ to $1396 \mathrm{PCU} / \mathrm{h}$ for P-section.In Figure 6, the stream speed may be observed fluctuating from 26 to $39 \mathrm{~km} / \mathrm{h}$ for S-section and 10 to $17 \mathrm{~km} / \mathrm{h}$ for P-section. On the other hand, three pedestrian parameters were primarily considered as design variables in the analysis viz. (a) pedestrian volume along the road 'Pal' (ped/h), (b) pedestrian volume across the road 'Pac' (ped/h) and (c) average lateral position of the pedestrian from the edge while walking along the road ' $D$ ' (m). Details of these pedestrian parameters observed at the P-section are given in Table 2. Furthermore, 
the complete dataset of traffic and pedestrian data for the P-section and the traffic data for the S-section are given in Appendix 1.

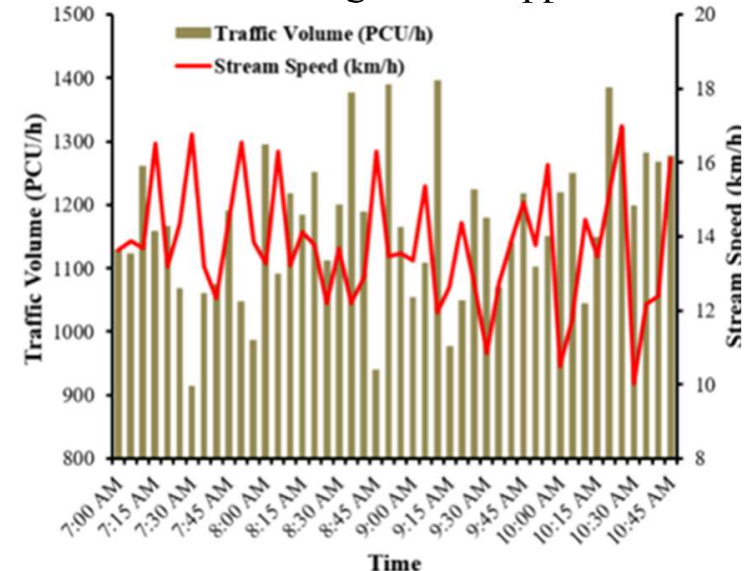

(a)

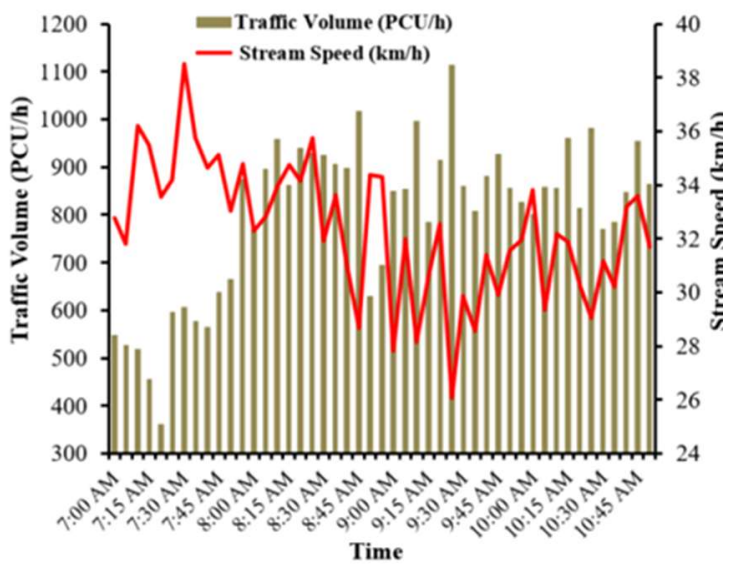

(b)

Figure 6: Variation in traffic volume and stream speed with time at a) standard and b) pedestrian section

Table 2: Details of pedestrian data observed at P-section

\begin{tabular}{lccccc}
\hline $\begin{array}{l}\text { Pedestrian } \\
\text { Parameters }\end{array}$ & Mean & $\begin{array}{l}\text { Standard } \\
\text { Deviation }\end{array}$ & Minimum & Maximum & $\begin{array}{l}\text { Coefficient } \\
\text { variation (\%) }\end{array}$ \\
\hline $\mathrm{P}_{\mathrm{al}}(\mathrm{ped} / \mathrm{h})$ & 806 & 133 & 492 & 1080 & 16 \\
$\mathrm{P}_{\mathrm{ac}}(\mathrm{ped} / \mathrm{h})$ & 112 & 47 & 24 & 240 & 42 \\
$\mathrm{D}(\mathrm{m})$ & 0.72 & 0.06 & 0.58 & 0.85 & 8.26 \\
\hline
\end{tabular}

\section{Speed prediction in absence of pedestrian movements}

Traffic speed and volume data for S-section for each 5-min time interval were considered to determine the relationship among macroscopic flow parameters viz. stream speed V $(\mathrm{km} / \mathrm{h})$, traffic density K (PCU/km) and traffic volume Q (PCU/h). Few popular macroscopic traffic flow models such as the Greenshields model(Greenshields, 1935), Greenberg model (Greenberg, 1959) and Underwood model (Underwood, 1961) were attempted to represent the dataset. The present study adopted the regression technique which revealed that Greenshields and Underwood models yielded better results $(0.78)$ in terms of goodness-of-fit as compared to Greenberg model. Statistical details of the Underwood model are provided in Table 3. Significance F corresponding to the model (Eqn. 2) developed using regression analysis was obtained as $3.92 \times 10^{-8}$ which is less than its critical value (0.05). It indicates the significance of the variable 'density' $(K)$ in determining the stream speed.

Table 3: Statistical details of stream speed model

\begin{tabular}{llllll}
\hline & $d f$ & $S S$ & $M S$ & $F$ & Significance F \\
\hline Regression & 1 & 0.1393 & 0.139347 & & \\
Residual & 48 & 0.1570 & 0.003272 & 42.5921 & $3.92 \mathrm{E}-08$ \\
Total & 49 & 0.2964 & & & \\
\hline
\end{tabular}

Hence, the present study adopted the Underwood model to represent the relationship between the stream speed and the density as given in Eqn. 2 . 


$$
\mathrm{V}=41.60 \mathrm{e}^{(-0.01 \mathrm{~K})}\left(\mathrm{R}^{2}=0.78\right)
$$

The model (Eqn. 2) can be useful to find the average stream speed if traffic density is known. However, the field estimation of traffic density is very challenging specifically for heterogeneous traffic conditions. In this regard, the traffic volume-based speed model is far more useful as the field estimation of traffic volume is comparatively easy. Therefore, using the fundamental relationship ofQ $=\mathrm{K} * \mathrm{~V}$, Eqn. 2 can be modified as

$$
V=41.60 e^{\left(-0.01 \frac{Q}{V}\right)}
$$

But, the only limitation associated with this model (Eqn. 3) is that the speed (V) lies on both sides of the equation. Therefore, the computation of ' $\mathrm{V}$ ' based on the traffic volume requires an iterative approach making the model computationally expensive and infeasible from the practical perceptive. It is easy to express ' $Q$ ' in terms of ' $V$ ' as given in Eqn. 4.

$$
\mathrm{Q}=100 \mathrm{~V} \ln \left(\frac{\mathrm{V}}{41.60}\right)
$$

However, with the objective to develop a volume-based speed model, the demand is to represent ' $\mathrm{V}$ ' as a function of ' $Q$ '.

To deal with this complexity, 'Lambert W function' was employed for estimating the speed under both congested and uncongested conditions. Suppose, $x$ and y relate to each other as

$$
y=f(x)=x \ln (x)
$$

and it is required to express ' $x$ ' in terms of ' $y$ '. Then,

$$
\mathrm{x}=\frac{\mathrm{y}}{\mathrm{W}(\mathrm{y})}
$$

Where the Lambert W function of ' $y$ ', W(y) can be computed as

$$
\begin{aligned}
& \mathrm{W}(\mathrm{y})=\sum_{\mathrm{n}=1}^{\infty} \frac{(-\mathrm{n})^{\mathrm{n}-1}}{\mathrm{n} !} \mathrm{y}^{\mathrm{n}} \\
& \mathrm{V}=\frac{-\mathrm{Q}}{100 \mathrm{~W}\left(\frac{-\mathrm{Q}}{4160}\right)}
\end{aligned}
$$

The model developed (Eqn. 8) will be useful to determine the stream speed based on observed traffic volume $(\mathrm{PCU} / \mathrm{h})$ in absence of on-street pedestrian movements. The value of Lambert $\mathrm{W}$ function can be calculated using any programming tool or can be taken directly from the Lambert W table (Biswas, Chandra and Ghosh, 2017).

\section{Impact of pedestrian movements on speed}

In order to assess the impact of pedestrian movements, the stream speed observed ( $\left.\mathrm{V}_{\text {observed }}\right)$ at $\mathrm{P}$-section was compared with the stream speed predicted $\left(\mathrm{V}_{\text {standard }}\right)$ at $\mathrm{S}$ - 
section using Eqn. 8 at the same traffic volume condition. Traffic volume data of each time interval at P-section were taken as inputs in Eqn. 8 which predicted the stream speeds $\left(\mathrm{V}_{\text {standard }}\right)$ in absence of pedestrian movements. To check whether there is a statistically significant difference between the stream speeds at two sections, the following hypothesizes were considered as given in Eqn. 9.

$$
\begin{aligned}
& \mathrm{H}_{0}: \mu_{d}=0 \\
& \mathrm{H}_{1}: \mu_{d} \neq 0
\end{aligned}
$$

Where, $\mu_{\mathrm{d}}$ is the mean of differences between stream speeds at two sections. To examine the hypothesis, a paired two sample t-test was performed. Results (as given in Table 4) indicate that the stream speed at P-section is significantly different from Ssection as $t_{\text {stat }}(37.28)$ exceeded its critical value of 2.01 with a level of significance of $5 \%$.

Table 4: Results of paired two sample t-test

\begin{tabular}{rllllll}
\hline S. No. & Variable & Mean & Variance & $\begin{array}{l}\text { Degree } \\
\text { offreedom }\end{array}$ & $t_{\text {stat }}$ & $\begin{array}{l}t_{\text {critical }} \\
\text { significance) }\end{array}$ \\
\hline 1. & $\begin{array}{l}\text { Stream speed at S- } \\
\text { section }\end{array}$ & 26.78 & 4.65 & 45 & 37.28 & 2.01 \\
2. & $\begin{array}{l}\text { Stream speed at P- } \\
\text { section }\end{array}$ & 13.75 & 2.73 & & & \\
\hline
\end{tabular}

Percent Speed Reduction (PSR) was determined by taking the relative percentage difference between two stream speeds as per Eqn. 10.

$$
\operatorname{PSR}(\%)=100\left(\frac{\mathrm{V}_{\text {standard }}-\mathrm{V}_{\text {observed }}}{\mathrm{V}_{\text {standard }}}\right)
$$

PSR was observed varying within a range of $29 \%$ to $62 \%$. This variation in PSR is attributed to the fact that the intensity of pedestrian movements at P-section was not constant rather it varied quite substantially as given in Table 2 . Hence, there was a need to model PSR considering pedestrian parameters as design variables. However, before that, the independency of these parameters among each other was essential to be examined. Pearson's correlation coefficient (as given in Eqn. 11) which is an indicator of collinearity existed between two variables, is commonly used for correlation check.

$$
\mathrm{r}_{\mathrm{x}, \mathrm{y}}=\frac{\sum\left(\mathrm{x}_{\mathrm{i}}-\overline{\mathrm{x}}\right)\left(\mathrm{y}_{\mathrm{i}}-\overline{\mathrm{y}}\right)}{\sqrt{\sum\left(\mathrm{x}_{\mathrm{i}}-\overline{\mathrm{x}}\right)^{2} \sum\left(\mathrm{y}_{\mathrm{i}}-\overline{\mathrm{y}}\right)^{2}}}
$$

Where, $r_{\mathrm{x}, \mathrm{y}}=$ Pearson's correlation coefficient of two variables $\mathrm{x}$ and $\mathrm{y}$ $\bar{x}, \bar{y}=$ sample mean of variable $\mathrm{x}$ and y respectively.

$r_{x, y}$ varies within the range of $[-1,1]$. Values of $r_{x, y}$ approaching closer to -1 and 1 indicate high 'negative' and 'positive' correlations respectively. On the other hand, a value of $\mathrm{r}_{\mathrm{x}, \mathrm{y}}$ nearby 0 indicates a low correlation and confirms independency of two input variables. The correlation matrix which provides the results of 'Pearson's correlation test' among different pedestrian parameters is given in Table 5.As it may be seen, all correlation coefficients are within a range of -0.14 to 0.28 which can be considered as weak correlation. Therefore, all the pedestrian parameters were considered together for further analysis. It is to be noted that the pedestrian parameters considered in this analysis are of diverse units and scales. 
Table 5: Correlation among design variables

\begin{tabular}{clll}
\hline Parameters & $P_{a l}$ & $P_{a c}$ & $D$ \\
\hline $\mathrm{P}_{\mathrm{al}}$ & 1.00 & - & - \\
$\mathrm{P}_{\mathrm{ac}}$ & -0.14 & 1.00 & - \\
$\mathrm{D}$ & 0.17 & 0.28 & 1.00
\end{tabular}

For this purpose, 'Feature scaling' technique was adopted to standardize these parameters using Eqn.12.

$$
\hat{\mathrm{z}}=\frac{\mathrm{z}_{\mathrm{i}}-z_{\min }}{\mathrm{z}_{\max }-z_{\min }}
$$

Where, $\hat{z}=$ standardized value of variable ' $\mathrm{z}$ '.

$\mathrm{Z}_{\mathrm{i}}=$ observed value of variable ' $\mathrm{z}$ '.

$Z_{\min }=$ minimum value of variable ' $z$ '.

$Z_{\max }=$ maximum value of variable ' $\mathrm{z}$ '

Minimum and maximum value of each pedestrian parameter was taken from Table 2. Standardized values of pedestrian parameters obtained after feature scaling became unit less and delimited within a common range of 0 to 1. Finally, a Percent Speed Reduction (PSR) model (Eqn.12) was developed by using multiple linear regression technique considering three standardized pedestrian parameters namely, a) pedestrian volume along the road 'Pal' (ped/h), (b) pedestrian volume across the road 'Pac' (ped/h) and (c) average lateral position of pedestrians from the edge while walking along the road ' $\hat{D}$ ' $(m)$. Statistical details of the multiple linear regression model are provided in Table 6. Significance F corresponding to the model (Eqn. 12) was obtained as $1.77 \times 10^{-21}$ which is less than its critical value (0.05). It indicates the significance of all input variables in determining the percent speed reduction (PSR).

Table 6: Statistical details of PSR model

\begin{tabular}{llllll}
\hline & $d f$ & $S S$ & $M S$ & $F$ & Significance F \\
\hline Regression & 3 & 99197.67 & 33065.89 & & \\
Residual & 43 & 10681.67 & 248.41 & 133.11 & $1.77 \mathrm{E}-21$ \\
Total & 46 & 109879.34 & & & \\
\hline
\end{tabular}

$$
\operatorname{PSR}(\%)=44.00 \widehat{\mathrm{Pal}_{\mathrm{l}}}+27.17 \widehat{\mathrm{P}_{\mathrm{ac}}}+19.33 \widehat{\mathrm{D}}\left(\mathrm{R}^{2}=0.90\right)
$$

The intercept of the empirical model (Eqn. 13) was kept as 'zero' intentionally so that there would be no reduction in the stream speed in the complete absence of pedestrian movements. It is also to be noted that the regression coefficients of all the pedestrian parameters are positive. It implies that an increase in any of these parameters will lead to an additional reduction in the stream speed. With every increase of $100 \mathrm{ped} / \mathrm{h}$ in pedestrian volume along and across the road will increase PSR by $7.48 \%$ and $20.37 \%$ respectively. Similarly, if the average lateral position of pedestrians from the edge shifts $0.1 \mathrm{~m}$ more towards the center of the road, PSR will increase by $16.30 \%$. This model (Eqn. 13 will be useful to predict 'percent speed reduction' of traffic stream under the influence of pedestrian movements with varying intensity. PSR as a performance measure could be a potential indicator of Level-of-Service (LOS) being provided to road users(Biswas and 
Pandey, 2020). In that way, this model can also be used to assess LOS on an urban street under the influence of pedestrian movements both along and across the road.

\section{Conclusions}

The present study forwards a novel methodology for predicting the stream speed under the influence of pedestrian movements. Some other novelties of the present research are as follows. The study has developed a'Lambert W function'-based speed prediction model for assessing the stream speed on two-lane undivided urban roads in the absence of any side-frictional activity. The model is quite unique in nature and removes additional complexities involved in obtaining the stream speed from an Underwood model. Further, this paper puts forward an empirical model predicting the percent speed reduction due to on-street pedestrian movements. The majority of the previous attempts were only focused on a particular pattern of pedestrian movements; either 'along the road' or 'across the road'. Also, these past studies considered 'pedestrian movements' as a binary variable; either 'present' or 'absent'. In this regard, the outcome of the present study is surely advancement over the former research as the developed model can estimate the combined impact of pedestrian movements both along and across the road with any given intensity. Moreover, the present study realized the importance of the lateral position of pedestrians while walking along the road in quantifying the impact on stream speed and therefore, introduced a new pedestrian parameter ' $\mathrm{D}$ ' to capture the lateral position aspect. Later, the parameter was proved to be significant in influencing the stream speed. This paper also demonstrates the field measurement procedure of this newly conceived parameter. This model or the methodology in general, will be helpful to transportation planners in estimating the efficiency of a pedestrian infrastructure project before implementation. This will add further clarity to decision-making on prioritizing alternative project proposals. Apart from this, some of the major findings of the investigation are summarized below.

Percent Speed Reduction (PSR) of vehicular traffic due to the presence of on-street pedestrian movements is not constant as it varies significantly due to the change in intensity of pedestrian parameters. Hence, this study yielded enough evidence that strongly recommends not to consider 'on-street pedestrian movements' merely as a categorical variable (either 'present' or 'absent') like previous studies while estimating its impact on the mobility of urban traffic.

'Pedestrian movements across the road' have more severe impact on the stream speed as compared to 'pedestrian movements along the road'. For instance, with every increase of $100 \mathrm{ped} / \mathrm{h}$ in pedestrian volume across the road, PSR is increased by $20.37 \%$ while it is only $7.48 \%$ in the case of pedestrians walking along the road. If the average lateral position of pedestrians shifts $0.1 \mathrm{~m}$ more towards the center of the carriageway, PSR increases by $16.30 \%$ as a subsequence.

This study examined the influence of pedestrian parameters on the stream speed at a specific road geometric condition (two-lane undivided road with a carriageway width of $5.5 \mathrm{~m}$ ). Hence, the use of the outcome of this study is strictly restricted to a particular category of road. Nonetheless, the methodology suggested in this paper can be taken up in future studies to address similar problems associated with other categories of road.

Appendix 1: Traffic and pedestrian data collected at S-section and P-section

\begin{tabular}{l|c|cc}
\hline \multirow{2}{*}{ Sl. No. } & S-section & \multicolumn{2}{c}{ P-section } \\
\cline { 2 - 3 } & Traffic data & Traffic data & Pedestrian data \\
\hline
\end{tabular}




\begin{tabular}{|c|c|c|c|c|c|c|c|}
\hline & $Q(P C U / h)$ & $V(\mathrm{~km} / \mathrm{h})$ & $Q(P C U / h)$ & $V(\mathrm{~km} / \mathrm{h})$ & $P_{a l}$ & $P_{a c}$ & $D$ \\
\hline 1 & 256.20 & 33.51 & 1129.08 & 13.63 & 672 & 204 & 0.7955 \\
\hline 2 & 64.80 & 33.64 & 1123.20 & 13.88 & 768 & 192 & 0.8336 \\
\hline 3 & 89.88 & 34.93 & 1261.20 & 13.68 & 852 & 168 & 0.7592 \\
\hline 4 & 546.48 & 32.79 & 1157.28 & 16.52 & 612 & 96 & 0.7118 \\
\hline 5 & 527.40 & 31.82 & 1165.68 & 13.20 & 780 & 120 & 0.6938 \\
\hline 6 & 517.20 & 36.21 & 1068.60 & 14.33 & 672 & 120 & 0.6973 \\
\hline 7 & 454.56 & 35.48 & 913.08 & 16.76 & 672 & 204 & 0.7955 \\
\hline 8 & 361.44 & 33.57 & 1060.80 & 13.19 & 744 & 48 & 0.6298 \\
\hline 9 & 595.80 & 34.21 & 1074.53 & 12.34 & 660 & 120 & 0.6300 \\
\hline 10 & 605.76 & 38.53 & 1190.88 & 14.36 & 804 & 120 & 0.7716 \\
\hline 11 & 577.08 & 35.75 & 1047.12 & 16.53 & 756 & 108 & 0.7508 \\
\hline 12 & 564.00 & 34.64 & 986.52 & 13.84 & 816 & 120 & 0.7926 \\
\hline 13 & 637.80 & 35.14 & 1295.16 & 13.28 & 708 & 120 & 0.6898 \\
\hline 14 & 664.32 & 33.03 & 1090.56 & 16.29 & 828 & 144 & 0.7732 \\
\hline 15 & 874.80 & 34.77 & 1216.92 & 13.21 & 876 & 120 & 0.7158 \\
\hline 16 & 769.08 & 32.28 & 1184.28 & 14.12 & 732 & 240 & 0.7213 \\
\hline 17 & 894.36 & 32.85 & 1250.76 & 13.77 & 1080 & 144 & 0.6661 \\
\hline 18 & 957.12 & 33.95 & 1110.96 & 12.19 & 900 & 120 & 0.7113 \\
\hline 19 & 862.20 & 34.75 & 1199.04 & 13.70 & 960 & 108 & 0.6875 \\
\hline 20 & 938.64 & 34.14 & 1375.68 & 12.18 & 696 & 84 & 0.7681 \\
\hline 21 & 928.20 & 35.77 & 1189.20 & 12.92 & 888 & 48 & 0.7209 \\
\hline 22 & 924.96 & 31.92 & 938.76 & 16.30 & 936 & 144 & 0.8038 \\
\hline 23 & 905.64 & 33.62 & 1389.72 & 13.47 & 768 & 168 & 0.6875 \\
\hline 24 & 897.96 & 31.01 & 1164.48 & 13.55 & 876 & 48 & 0.7384 \\
\hline 25 & 1017.48 & 28.69 & 1054.08 & 13.35 & 1008 & 72 & 0.7202 \\
\hline 26 & 628.20 & 34.39 & 1108.44 & 15.36 & 936 & 36 & 0.7051 \\
\hline 27 & 693.60 & 34.29 & 1395.84 & 11.96 & 936 & 72 & 0.6769 \\
\hline 28 & 849.84 & 27.80 & 976.44 & 12.63 & 600 & 120 & 0.5830 \\
\hline 29 & 852.36 & 32.00 & 1048.20 & 14.36 & 1032 & 96 & 0.6843 \\
\hline 30 & 995.28 & 28.14 & 1224.56 & 12.67 & 705 & 48 & 0.6221 \\
\hline 31 & 784.08 & 30.63 & 1178.04 & 10.86 & 696 & 84 & 0.5879 \\
\hline 32 & 914.40 & 32.56 & 1070.38 & 12.76 & 912 & 120 & 0.7309 \\
\hline 33 & 1114.08 & 26.07 & 1140.24 & 13.88 & 888 & 132 & 0.8473 \\
\hline 34 & 859.68 & 29.87 & 1217.64 & 14.92 & 756 & 96 & 0.7333 \\
\hline 35 & 806.28 & 28.57 & 1102.20 & 13.75 & 888 & 48 & 0.6986 \\
\hline 36 & 880.32 & 31.41 & 1149.24 & 15.93 & 792 & 144 & 0.7417 \\
\hline 37 & 927.12 & 29.89 & 1218.24 & 10.48 & 588 & 24 & 0.7677 \\
\hline 38 & 854.64 & 31.56 & 1249.92 & 11.76 & 912 & 60 & 0.7920 \\
\hline 39 & 825.48 & 31.95 & 1043.64 & 14.45 & 756 & 192 & 0.7159 \\
\hline 40 & 801.72 & 33.84 & 1147.56 & 13.47 & 648 & 84 & 0.7537 \\
\hline 41 & 856.56 & 29.36 & 1384.56 & 15.21 & 876 & 96 & 0.7158 \\
\hline 42 & 854.76 & 32.20 & 1321.32 & 16.98 & 492 & 132 & 0.6439 \\
\hline 43 & 959.40 & 31.87 & 1198.68 & 10.02 & 840 & 108 & 0.6521 \\
\hline 44 & 812.52 & 30.24 & 1281.24 & 12.18 & 936 & 108 & 0.6981 \\
\hline 45 & 981.84 & 29.07 & 1267.32 & 12.39 & 1068 & 108 & 0.7663 \\
\hline 46 & 770.28 & 31.17 & 1276.56 & 16.11 & 756 & 96 & 0.7333 \\
\hline 47 & 784.80 & 30.20 & 1129.08 & 13.63 & 672 & 204 & 0.7955 \\
\hline 48 & 846.72 & 33.19 & 1123.20 & 13.88 & 768 & 192 & 0.8336 \\
\hline
\end{tabular}

\section{References}

Adinarayana, I. and Anil, N. C. (2017) 'The Study Exploration towards Side Friction Influences by traffic performance measures on roads', 5(11).

Advani, M. and Nisha, G. (2013) 'Behavioural analysis of pedestrians for walking on footpath and on carriageway in "space-sharing" traffic scenario', Indian Highways, Indian Roads Congress, New Delhi, 41(7), pp. 40-46. 
Aronsson, K. F. M. and Bang, K. L. (2006) 'Influence of vehicle speed profiles of interactions with other road users', European Transport and Contributors, pp. 1-14.

Biswas, S., Chandra, S. and Ghosh, I. (2017) 'Use of Lambert W function in determining speed for macroscopic traffic flow models', European Transport, 63(3), pp. 1-10.

Biswas, S. and Pandey, A. (2020) 'Development of "Speed Ratio" Based Level of Service Criteria on Undivided Urban Streets in Mixed Traffic Context', Canadian Journal of Civil Engineering. doi: https://doi.org/10.1139/cjce-2020-0104.

Chen, Q. and Wang, Y. (2015) 'Cellular automata (CA) simulation of the interaction of vehicle flows and pedestrian crossings on urban low-grade uncontrolled roads', Physica A: Statistical Mechanics and its Applications, 432, pp. 43-57. doi: 10.1016/j.physa.2015.03.004.

Corazza, M. V., Di Mascio, P. and Moretti, L. (2018) 'Management of sidewalk maintenance to improve walking comfort for senior Citizens', WIT Transactions on the Built Environment, 176, pp. 195-206. doi: 10.2495/UT170171.

CSIR- Central Road Research Institute (2017) Indian Highway Capacity Manual (IndoHCM). New Delhi, India.

Golakiya, H. D. and Dhamaniya, A. (2019) 'Modeling Speed and Capacity Estimation at Urban Midblock Sections under the Influence of Crossing Pedestrians', 145(1998), pp. 1-14. doi: 10.1061/JTEPBS.0000260.

Greenberg, H. (1959) 'An analysis of traffic flow', Operations Research, 7(1), pp. 7985.

Greenshields, B. D. (1935) 'A study of traffic capacity', Highway Research Board Proceedings, pp. 448-477.

Hawas, Y. E. and Khan, M. B. (2012) 'A fuzzy logic modeling approach to assess the speed limit suitability in urban street networks', in International Conference on Agents and Artificial Intelligence, ICAART 2012. Vilamoura, Algarve, Portugal, pp. 1-16. doi: 10.1007/978-3-642-36907-0.

IRC (1990) 'I.R.C. (1990) 106. Guidelines for Capacity of Urban Roads in Plain Areas', Indian Highways, Indian Roads Congress, New Delhi, pp. 1-10.

Jin, S. et al. (2013) 'Dynamic characteristics of traffic flow with consideration of pedestrians' road-crossing behavior', Physica A: Statistical Mechanics and its Applications, 392(18), pp. 3881-3890. doi: 10.1016/j.physa.2013.04.030.

Kadali, B. R., Chiranjeevi, T. and Rajesh, R. (2015) 'Effect of pedestrians un-signalized mid-block crossing on vehicular speed', International Journal for Traffic and Transport Engineering, 5(2), pp. 170-183. doi: 10.7708/ijtte.2015.5(2).07.

Kadali, R. B. and Tadi, C. (2015) 'EFFECT OF PEDESTRIANS UN-SIGNALIZED MID-BLOCK CROSSING ON EFFECT OF PEDESTRIANS UN-SIGNALIZED MID-BLOCK CROSSING ON VEHICULAR SPEED', 5(June). doi: 10.7708/ijtte.2015.5(2).07.

Marinelli, G., Dalmazzo, D. and Bassani, M. (2013) 'Variables influencing speed distribution on urban arterials and collectors', (January).

Munawar, A. (2011) 'Speed and Capacity for Urban Roads, Indonesian Experience', Procedia - Social and Behavioral Sciences, 16, pp. 382-387. doi: 10.1016/j.sbspro.2011.04.459.

Salini, S., George, S. and Ashalatha, R. (2016) 'Effect of Side Frictions on Traffic Characteristics of Urban Arterials', Transportation Research Procedia, 17(December 2014), pp. 636-643. doi: 10.1016/j.trpro.2016.11.118.

Shaaban, K., Muley, D. and Mohammed, A. (2018) 'Analysis of illegal pedestrian 
crossing behavior on a major divided arterial road', 54, pp. 124-137.

Sukhadia, H. et al. (2016) 'The Effect of Events on Pedestrian Behavior and its Comparison with Normal Walking Behavior in CBD Area in Indian Context', Transportation Research Procedia, 17(December 2014), pp. 653-663. doi: 10.1016/j.trpro.2016.11.120.

Thiessen, A., El-Basyouny, K. and Gargoum, S. (2017) 'Operating Speed Models for Tangent Segments on Urban Roads', Transportation Research Record, 2618(1), pp. 91-99. doi: 10.3141/2618-09.

Underwood, R. T. (1961) 'Speed, volume, and density relationships', Yale Bureau of Highway Traffic, pp. 141-188.

Várhelyi, A. (1996) Dynamic speed adaptation based on information technology, a theoretical background. Lund Institute of Technology, Lund University, Sweden. 